\title{
TREINAMENTO MUSCULAR INSPIRATÓRIO EM INDIVÍDUOS OBESOS: um estudo piloto
}

\section{INSPIRATORY MUSCLE TRAINING IN OBESE INDIVIDUALS:}

\author{
a pilot study
}

\author{
Letícia Donato', Loani Maldaner', Roberta Cattaneo Horn², Leandro Chaves ${ }^{3}$, \\ Leandro de Moraes Kohl'4, Isabella Martins de Albuquerque ${ }^{5}$, \\ Gabriela Tassotti Gelatti ${ }^{6}$, Carine Cristina Callegaro7
}

\section{RESUMO}

Investigar os efeitos do TMI sobre a lipoperoxidação em indivíduos obesos. Metodologia: Participaram desse estudo sete indivíduos obesos com idade média de $34 \pm 5$ anos. Foi avaliada a força muscular respiratória, a resistência muscular inspiratória, a tolerância ao exercício pelo teste de caminhada de 6 minutos (TC6m), a qualidade de vida e a ocorrência de lipoperoxidação através da determinação dos níveis de substâncias reativas ao ácido tiobarbitúrico (TBARS), antes e após quatro semanas de TMI, realizado à $30 \%$ da pressão inspiratória máxima. Resultados: O TMI induziu aumento na força muscular inspiratória ( $p=0,008)$, na resistência muscular inspiratória $(p=0,0003)$, bem como ocasionou melhora no domínio físico do escore de qualidade de vida. Entretanto, o TMI não alterou a distância percorrida no TC6m e os níveis de TBARS $(p=0,85)$. Conclusão: Quatro semanas de TMI aumentam a força e resistência muscular inspiratória, bem como melhoram o domínio físico da qualidade de vida, porém não altera os níveis de TBARS em obesos.

Descritores: Obesidade; Respiração; Espécies Reativas de Oxigênio; Estresse Oxidativo; Exercício.

\section{ABSTRACT}

To investigate the effects of TMI on lipid peroxidation in obese individuals. Methods: Seven obese subjects with a mean age of $34 \pm 5$ years participated in this study. It was evaluated the respiratory muscle strength, the inspiratory muscle endurance, the exercise tolerance by the 6-minute walk test (6MWT) and the quality of life, as well, is was investigate the occurrence of lipid peroxidation by determining the levels of reactive substances thiobarbituric acid (TBARS) before and after four weeks of TMI, performed at $30 \%$ of maximal inspiratory pressure. Results: TMI induced increase in inspiratory muscle strength $(p=0.008)$, inspiratory muscle resistance $(p=0.0003)$ and improvement in the physical domain of quality of life score. However, TMI did not alter the distance on the 6MWT and the levels of TBARS ( $p=0.85$ ). Conclusion: Four weeks of TMI increases inspiratory muscle strength and endurance and improves the physical domain of quality of life, but does not change the TBARS levels in obese.

Descriptors: Obesity; Breathing; Reactive Oxygen Species; Oxidative Stress; Exercise.
${ }^{1}$ Graduada em Fisioterapia pela Universidade de Cruz Alta (UNICRUZ), Cruz Alta, RS, Brasil.

${ }^{2}$ Doutora em Ciências Biológicas: Bioquímica Toxicológica pela Universidade Federal de Santa Maria (UFSM), Santa Maria, RS, Brasil.

${ }^{3}$ Acadêmico de Biomedicina pela Universidade de Cruz Alta (UNICRUZ), Cruz Alta, RS, Brasil.

${ }^{4}$ Mestre em Ciências da Saúde: Cardiologia pelo Instituto de Cardiologia do Rio Grande do Sul/Fundação Universitária de Cardiologia, Porto Alegre, RS, Brasil

${ }^{5}$ Docente do Departamento de Fisioterapia e Reabilitação da Universidade Federal de Santa Maria (UFSM), Santa Maria, RS, Brasil.

${ }^{6}$ Mestranda do Programa de PósGraduação em Atenção Integral à Saúde (UNICRUZ), Cruz Alta, $\mathrm{RS}$, Brasil.

${ }^{7}$ Doutora em Ciências Cardiovasculares pela Universidade Federal do Rio Grande do Sul (UFRGS), Porto Alegre, RS, Brasil. 


\section{Introdução}

A expectativa de vida vem crescendo na população mundial, sendo acompanhada pelo aumento da incidência de problemas de saúde pública como, por exemplo, a obesidade. Dados epidemiológicos da Organização Mundial de Saúde estimam que mais de 100 milhões de adultos estejam com sobrepeso e 500 milhões deles apresentam diagnóstico de obesidade 1.

No Brasil, dados recentes do Ministério da Saúde ${ }^{2}$ apontam que houve um crescimento da incidência de excesso de peso de $23 \%$ nos últimos nove anos. Embora o percentual de obesidade (17,9\% em 2014) não tenha sofrido alteração nos últimos anos, constitui fator de risco para doenças crônicas, responsáveis por mais de $70 \%$ dos óbitos no Brasil. Esses dados apresentam grande relevância para estruturação de programas de saúde pública, pois além de representar risco de mortalidade, a obesidade está relacionada com alterações respiratórias ${ }^{3,4}$ e ocorrência de estresse oxidativo que podem contribuir para o desenvolvimento de doenças cardiovasculares ${ }^{5}$.

A presença de tecido adiposo em excesso na obesidade contribui para o aumento na formação de espécies reativas, tendo em vista que os pré-adipócitos e adipócitos são identificados como uma fonte de citocinas pró-inflamatórias, incluindo fator de necrose tumoral-a, interleucina-1 e interleucina-6. Um aumento na concentração de citocinas pode ser responsável pelo aumento do estresse oxidativo, pois elas estimulam a produção de espécies reativas de oxigênio e nitrogênio por macrófagos e monócitos ${ }^{6}$. $O$ tecido adiposo também tem a capacidade de secretar angiotensina II, 0 qual estimula a nicotinamida adenina dinucleótido fosfato oxidase, sendo que esta compreende a principal via para a produção de espécies reativas de oxigênio (EROs) em adipócitos ${ }^{7}$.

0 treinamento muscular inspiratório (TMI) apresenta benefícios para indivíduos obesos, assim como ganho de força muscular inspiratória, melhora da mobilidade torocoabdominal ${ }^{8}$ e aumento da capacidade funcional ${ }^{9}$. Além disso, estudos mostram que o TMl acarreta benefícios em diversas condições patológicas, como redução da dispneia e melhora a tolerância ao exercício na fibrose cística ${ }^{10}$; assim como melhora a função respiratória na doença pulmonar obstrutiva crônica (DPOC) $)^{11}$. No entanto, os dados sobre os efeitos do TMI sobre a peroxidação lipídica em indivíduos obesos ainda são escassos. Sendo assim, o objetivo desse estudo foi investigar os efeitos do TMI sobre a força muscular respiratória, resistência muscular inspiratória, qualidade de vida e sobre a peroxidação lipídica de indivíduos obesos.

\section{Metodologia}

O estudo se caracteriza por uma série de casos. A amostra foi selecionada por conveniência sendo composta por sete indivíduos obesos, cinco do gênero feminino e dois do gênero masculino, os quais apresentavam idade entre 28 a 40 anos, com peso entre 76 a $128 \mathrm{~kg}$. Foram excluídos os indivíduos com doença arterial coronariana, doença pulmonar, diabetes mellitus e doenças metabólicas. Todos os voluntários foram submetidos à coleta de sangue venoso $(5 \mathrm{~mL})$ para a realização dos exames laboratoriais de glicose de jejum, triglicerídeos, lipoproteínas de baixa densidade (LDL) (calculado pela fórmula de Friedewald) e lipoproteínas de alta densidade (HDL) de acordo com o protocolo dos Kits da marca Labtest ${ }^{\circledR}$, no Laboratório de Análises Clínicas da Universidade de Cruz Alta.

Todos os voluntários assinaram o Termo de Consentimento Livre e Esclarecido (TCLE) aprovado pelo Comitê de Ética em Pesquisa da Universidade de Cruz Alta com CAAE nº 0023.0.417.000-11.

\section{Avaliações}

Após 15 minutos de repouso, os voluntários foram submetidos à verificação da pressão arterial realizada através da técnica auscultatória por meio de esfigmomanômetro de coluna de mercúrio (Heidji, Press Control Indústria e Comércio LTDA, Brasil) conforme a VI Diretrizes Brasileiras de Hipertensão ${ }^{12}$. A frequência cardíaca de repouso foi aferida por meio de oxímetro de pulso (CONTEC, CMS50C, Brasil ) posicionado no dedo médio.

Força muscular respiratória: A força muscular inspiratória e expiratória foi determinada pela mensuração da pressão inspiratória máxima (Pimáx) e pressão expiratória máxima (Pemáx) através de um manovacuômetro analógico (Famabras, Brasil) , calibrado em $\mathrm{cmH}_{2} \mathrm{O}$ com limite operacional de $+/-150 \mathrm{cmH}_{2} \mathrm{O}$, conectado a um sistema composto por 
duas válvulas unidirecionais acopladas a um bucal. As manobras respiratórias foram repetidas até encontrar seis medidas com variação menor que $10 \%$. O maior valor obtido foi utilizado como Pimáx e como Pemáx ${ }^{13}$ conforme recomendação da American Thoracic Society ${ }^{14}$.

Resistência muscular inspiratória: O teste de resistência com carga progressiva dos músculos inspiratórios foi realizado com o paciente respirando continuamente através de um bucal conectado a uma resistência linear (Threshold Inspiratory Muscle Trainer, Healthscan Products Inc. Cedar Grove, New Jersey, USA) de 50\% Pimáx com incremento de $10 \%$ da Pimáx a cada três minutos até o momento em que o paciente foi incapaz de continuar o teste. O maior valor de pressão inspiratória sustentada por no mínimo 60 segundos e expressa como percentual da Pimáx foi utilizada como medida da resistência muscular inspiratória ${ }^{15}$.

Tolerância ao exercício: 0 teste de caminhada de seis minutos (TC6m) foi utilizado para estimar a tolerância ao exercício. Os indivíduos foram orientados a caminhar em uma superfície plana de 30 metros durante seis minutos, com demarcação da distância total percorrida pelo indivíduo, de acordo com as normas da American Thoracic Society ${ }^{16}$.

Qualidade de vida: A qualidade de vida foi avaliada por meio do questionário WOQOL-Bref, o qual apresenta 26 questões que abrangem capacidade funcional; aspectos físicos; dor; estado geral de saúde; vitalidade; aspectos sociais; aspecto emocional e saúde mental, além da pontuação total, a qual considera a média aritmética de todos os domínios ${ }^{17}$.

Peroxidação lipídica: A peroxidação lipídica foi avaliada através da determinação dos níveis plasmáticos de substâncias reativas ao ácido tiobarbitúrico (TBARS) de acordo com o método descrito por Jentzsch et al. ${ }^{18}$. Baseado na reação do malondialdeido (MDA) com o ácido tiobarbitúrico (TBA), que tem como produto um composto de coloração rosa, que pode ser medido em espectrofotômetro (BEL Engineering, modelo V-M5 espectrofotômetro visível, Itália) visível a $532 \mathrm{~nm}$. Os resultados foram expressos como por nmol MDA/mL.

\section{$\underline{\text { Intervenção }}$}

O TMI foi realizado através de um equipamento ajustado para fornecer uma resistência linear à inspiração (Threshold Inspiratory Muscle Trainer, Healthscan Products Inc, Cedar Grove, New Jersey) de 30\% Pimáx, realizado durante 30 minutos, cinco vezes por semana durante quatro semanas ${ }^{15}$. A Pimáx foi mensurada uma vez por semana para ajustar a resistência inspiratória em $30 \%$ da Pimáx. As sessões de treinamento muscular inspiratório foram realizadas no domicílio do sujeito, sendo sempre supervisionadas pelos pesquisadores. Durante o TMI foi utilizada a escala de dispnéia e esforço de Borg ${ }^{19}$ no início e ao final das sessões. Ao final do período de treinamento as avaliações foram repetidas.

\section{Análise estatística}

Os dados foram expressos através de média e desvio padrão. Os efeitos do treinamento muscular inspiratório sobre a função muscular respiratória, qualidade de vida e peroxidação lipídica foram analisados através do Teste $t$ de Student para amostras pareadas. A associação entre as variáveis foram analisadas através da correlação de Pearson. Um valor $p<0,05$ foi considerado significativo.

\section{Resultados}

A Tabela 1 apresenta as características da amostra. Seis indivíduos apresentaram Índice de Massa Corporal (IMC) $>30$ e $<40 \mathrm{~kg} / \mathrm{m}^{2}$ (obesidade de grau 2 segundo critérios da Organização Mundial de Saúde) e um indivíduo apresentou IMC $>40 \mathrm{~kg} / \mathrm{m}^{2}\left(40,5 \mathrm{~kg} / \mathrm{m}^{2}\right)$ sendo este classificado como obesidade grau 3 (obesidade mórbida) ${ }^{20}$. Os indivíduos obesos apresentaram perfil lipídico, bem como índices glicêmicos de jejum considerados normais. Na Tabela 2 pode-se constatar que o TMI promoveu um incremento da força muscular inspiratória, representado pelo aumento da Pimáx e pelo aumento do percentual da Pimáx predita para o sexo e para a idade. Além disso, observou-se um aumento da resistência muscular inspiratória, demonstrado pela maior duração e aumento da pressão inspiratória tolerada durante o teste de resistência dos músculos inspiratórios com carga progressiva. Já a Pemáx e o percentual da Pemáx predita para o sexo e a idade não diferiram estatisticamente após o programa de TMI. 
Tabela 1 - Características dos indivíduos

\begin{tabular}{c|c}
\hline \multicolumn{2}{c}{ Obesos (n=7) } \\
\hline Idade (anos) & $34 \pm 5$ \\
\hline Peso (kg) & $96 \pm 17$ \\
\hline Altura (metros) & $1,62 \pm 0,08$ \\
\hline IMC (kg/m $\mathbf{)}$ & $36 \pm 3$ \\
\hline Glicose (mg/dL) & $84 \pm 9$ \\
\hline Triglicerideos (mg/dL) & $95 \pm 48$ \\
\hline Colesterol total (mg/dL) & $142 \pm 36$ \\
\hline HDL (mg/dL) & $43 \pm 13$ \\
\hline LDL (mg/dL) & $80 \pm 40$ \\
\hline PAS (mmHg) & $127 \pm 13$ \\
\hline PAD (mmHg) & $73 \pm 28$ \\
\hline FC (bpm) & $83 \pm 15$ \\
\hline
\end{tabular}

Dados apresentados através de média e desvio padrão. Índice de Massa Corporal (IMC); Colesterol de Alta Densidade (HDL); Colesterol de Baixa Densidade (LDL);

Pressão Arterial Sistólica (PAS); Pressão Arterial Diastólica (PAD); Frequência Cardíaca (FC).

Neste estudo não houve associação entre Pimáx e IMC pré $(r=-0,12 ; p=0,79)$ e pós-treinamento muscular inspiratório $(r=0,16 ; p=0,71)$, assim como não houve associação estatisticamente significativa entre IMC e a duração do teste de resistência muscular inspiratória com carga progressiva pré $(r=0,60 ; p=0,15)$ e pós-treinamento da musculatura inspiratória $(r=0,29 ; p=0,51)$.

Apesar do aumento na força muscular inspiratória ocorrido após o TMI, não foi observada alteração nos níveis de TBARS (pré-treinamento $=11 \pm 11 \mathrm{nmol} \mathrm{MDA} / \mathrm{mL}$; pós-treinamento $=13 \pm 11 \mathrm{nmol} \mathrm{MDA} / \mathrm{mL}, p=0,85$ ).

Tabela 2 - Efeitos do Treinamento Muscular Inspiratório sobre a Função Muscular Respiratória

\begin{tabular}{c|c|c|c}
\hline & $\begin{array}{c}\text { Pré-treinamento } \\
(\mathbf{n = 7})\end{array}$ & $\begin{array}{c}\text { Pós-treinamento } \\
(\mathbf{n = 7})\end{array}$ & P * \\
\hline Pimáx (cmH $\mathbf{2})$ & $99 \pm 26$ & $127 \pm 28$ & 0,008 \\
\hline \% Pimáx predita & $95 \pm 18$ & $124 \pm 28$ & 0,008 \\
\hline Pemáx (cmH_o) & $110 \pm 24$ & $121 \pm 19$ & 0,14 \\
\hline \% Pemáx predita & $103 \pm 15$ & $117 \pm 18$ & 0,10 \\
\hline $\begin{array}{c}\text { Pimáx - teste- } \\
\text { incremental }\end{array}$ & $73 \pm 20$ & $114 \pm 26$ & 0,001 \\
\hline $\begin{array}{c}\text { \% Pimáx - teste- } \\
\text { incremental/Pimáx }\end{array}$ & $74 \pm 8$ & $89 \pm 4$ & 0,002 \\
\hline $\begin{array}{c}\text { Duração - teste- } \\
\text { incremental (minutos) }\end{array}$ & $11,9 \pm 1,6$ & $15,7 \pm 1,6$ & 0,0003 \\
\hline
\end{tabular}

Pimáx (Pressão Inspiratória Máxima); \%Pimáxpred (Percentual da Pressão Inspiratória Máxima predita para sexo e idade); Pemáx (Pressão Expiratória Máxima); \%Pemáxpred (Percentual da Pressão Expiratória Máxima predita para sexo e idade); Pimáx - teste-incremental (Pressão Inspiratória Máxima obtida no Teste de Resistência dos Músculos Inspiratórios com Carga Progressiva).

* Teste t de Student pareado 
Em relação à qualidade de vida, o TMI induziu um aumento significativo no domínio físico do questionário WOQOL-Bref $(p=0,01)$, enquanto que houve apenas uma tendência de melhora no domínio psicológico, o qual não atingiu significância estatística. Já os domínios social e ambiental não diferiram estatisticamente após o TMI. No aspecto qualidade de vida geral houve uma tendência de melhora após as quatro semanas do TMI (Tabela 3).

No que diz respeito à distância percorrida no TC6m, nossos resultados demonstraram que o TMI não alterou a distância percorrida durante 0 teste de caminhada de seis minutos (pré-treinamento $=503 \pm 104$ metros vs. póstreinamento $=465 \pm 111$ metros, $p=0,39$ ), mostrando que quatro semanas de TMI não afetou a tolerância ao exercício em indivíduos obesos.

Tabela 3 - Efeitos do treinamento muscular inspiratório sobre a qualidade de vida determinada pelo instrumento WHOCOL-Bref.

\begin{tabular}{c|c|c|c}
\hline $\begin{array}{c}\text { Escores de qualidade } \\
\text { de vida }\end{array}$ & $\begin{array}{c}\text { Pré-treinamento } \\
(\mathbf{n}=\mathbf{7})\end{array}$ & $\begin{array}{c}\text { Pós-treinamento } \\
(\mathbf{n = 7})\end{array}$ & $\boldsymbol{P}^{*}$ \\
\hline Domínio I- Físico & $69 \pm 17$ & $80 \pm 19$ & 0,01 \\
\hline Domínio II- Psicológico & $60 \pm 14$ & $68 \pm 17$ & 0,06 \\
\hline Domínio III- Social & $73 \pm 10$ & $73 \pm 20$ & 1,0 \\
\hline Domínio IV- Ambiente & $58 \pm 11$ & $66 \pm 13$ & 0,16 \\
\hline Qualidade de Vida Geral & $65 \pm 10$ & $72 \pm 13$ & 0,09 \\
\hline
\end{tabular}

* Teste t de Student pareado

\section{Discussão}

Nesse estudo demonstrou-se que quatro semanas de treinamento muscular inspiratório foram insuficientes para induzir alterações na peroxidação lipídica, apesar dos efeitos benéficos observados sobre a força e resistência muscular inspiratória, bem como sobre o domínio físico da qualidade de vida em indivíduos obesos.

No presente estudo, o TMI falhou em induzir alterações no estresse oxidativo semelhante aos achados do trabalho de Vincent et al. ${ }^{21}$, no qual o treinamento físico de resistência, realizado durante seis meses, não modificou os valores de TBARS de repouso, mas reduziu o TBARS entre 14\% e 18\% apenas após teste de ergométrico. Entretanto, outros estudos envolvendo o treinamento físico demonstram achados divergentes ${ }^{22-25}$. Um estudo em modelo animal demonstrou que um mês de treinamento aeróbico aumenta os níveis de TBARS ${ }^{22}$. Da mesma forma, Gounchar ${ }^{23}$ constatou que 0 treinamento de alta intensidade realizado durante um mês foi acompanhado pelo aumento dos níveis de TBARS ${ }^{24}$.

A intensidade do TMI também poderia influenciar o estresse oxidativo como evidenciado previamente através do treinamento de natação, que induziu aumento nos níveis de TBARS circulante, reforçando a teoria de que o exercício realizado em intensidade de transição metabólica aeróbia/anaeróbia pode gerar estresse oxidativo em ratos ${ }^{25}$. Esses achados foram reforçados por outro estudo que demonstrou que os níveis de TBARS foram elevados no sangue dos animais treinados durante quatro semanas em comparação aos controles, indicando maior dano oxidativo ${ }^{26}$. Em contraste, um estudo mais recente mostrou que o treinamento em esteira ergométrica, durante dois meses, não alterou os níveis de TBARS $^{27}$. Da mesma forma, ratos treinados através de um programa de natação não apresentaram diferenças nos níveis de peroxidação lipídica no músculo esquelético ${ }^{28}$. Os efeitos do treinamento físico poderiam divergir dos efeitos do treinamento muscular inspiratório, no entanto, a carência de estudos sobre os efeitos do treinamento muscular inspiratório permite apenas especulações a esse respeito.

Apesar do treinamento muscular inspiratório não induzir alterações na peroxidação lipídica, nesse estudo demonstrouse que apenas quatro semanas de treinamento efetivamente eleva a força muscular inspiratória. Esse achado vai ao encontro de estudos publicados previamente envolvendo indivíduos obesos submetidos à reeducação funcional respiratória que apresentaram aumento da Pimáx de $64 \pm 11 \mathrm{cmH}_{2} \mathrm{O}$ para $92 \pm 31 \mathrm{cmH}_{2} \mathrm{O}^{8}$. Esse aumento de força muscular inspiratória foi semelhante ao observado neste estudo, onde a Pimáx aumentou de $99 \pm 26 \mathrm{cmH}_{2} \mathrm{O}$ para $127 \pm 28 \mathrm{cmH}_{2} \mathrm{O}$. Além disso, observou-se uma melhora significativa na Pimáx de $45 \pm 30 \%$ em remadores do gênero feminino altamente treinados e submetido ao $\mathrm{TMI}^{29}$. Já em pacientes cronicamente doentes, como na fibrose cística, os valores de Pimáx após o TMI podem 
aumentar em até $80 \%{ }^{30}$. Ainda, em adultos e crianças asmáticos ${ }^{31}$, bem como em pacientes com DPOC ${ }^{32}$, o TMl aumentou os valores de Pimáx e Pemáx, o que diverge dos achados do presente estudo em que não houve alterações significativas na Pemáx, possivelmente devido a especificidade do treinamento muscular inspiratório.

Hipotetiza-se no presente estudo que o período de TMI pode ter sido insuficiente para promover adaptações das fibras musculares inspiratórias que modifiquem a tolerância ao exercício ${ }^{13}$. Por exemplo, um programa de TMI de seis semanas não alterou a distância percorrida no TC6m em pacientes com DPOC ${ }^{33}$, enquanto que um programa realizado durante 12 semanas em pacientes com insuficiência cardíaca, resultou em um aumento de $19 \%$ na distância percorrida ${ }^{15}$. Dados semelhantes ao último estudo foram encontrados em pacientes com doença renal crônica ${ }^{34}$.

Interessantemente, o aumento da força muscular inspiratória induzido pelo treinamento muscular inspiratório melhora a qualidade de vida de indivíduos obesos, conforme demonstrado através do questionário WOQOL-Bref, independente de modificação na tolerância ao exercício. Os achados da melhora da qualidade de vida após o TMl em obesos estão de acordo com estudos prévios realizados em pacientes com insuficiência cardíaca ${ }^{15}$ e DPOC ${ }^{35}$. Especificamente em nosso estudo, a melhora da qualidade de vida está relacionada com o domínio físico, visto que não foi observada nenhuma mudança significativa nos domínios social e ambiente, provavelmente devido ao fato do programa não ter sido realizado em grupo.

Finalmente, é importante mencionar as limitações do presente estudo: $1^{\circ}$ ) a maioria dos indivíduos envolvidos em nosso estudo apresentavam obesidade grau 2. Dessa forma, os achados desse estudo não podem ser aplicados a indivíduos portadores de obesidade mórbida. Especula-se que indivíduos com obesidade mórbida ou associada à síndrome metabólica poderiam ser mais beneficiados com o TMI do que a amostra do presente estudo; $2^{\circ}$ ) o TMI parece apresentar maiores benefícios em indivíduos com fraqueza muscular inspiratória, no entanto nossa amostra não apresentava fraqueza muscular inspiratória; $3^{\circ}$ ) nesse estudo não foi controlada a dieta, o que poderia influenciar os níveis de peroxidação lipídica; e $4^{\circ}$ ) o tamanho amostral de nosso estudo pode ter sido insuficiente para detectarmos uma associação significativa entre IMC e resistência muscular inspiratória.

Estudos controlados e randomizados com maior tamanho amostral poderiam ser realizados com o objetivo de confirmar nossos achados, incluindo outros marcadores de estresse oxidativo, bem como de defesas antioxidantes.

\section{Considerações Finais}

A partir deste estudo pode-se concluir que o TMI não altera os níveis de TBARS, mas apresenta efeitos benéficos como o aumento da força e resistência muscular inspiratória, além da melhora no domínio físico da qualidade de vida em indivíduos obesos.

\section{Referências}

1. Babb TG. Obesity: challenges to ventilatory control during exercise a brief review. Respir Physiol Neurobiol. 2013; 189(2):364-70.

2. Ministério da Saúde, Vigitel 2014: Obesidade estabiliza no Brasil, mas excesso de peso aumenta. Data de Cadastro: 15/04/2015 as 18:04:11 alterado em 16/04/2015 as 09:04:51.

3. Koenig SM. Pulmonary complications of obesity. Am J Med Sci. 2001; 321(4):249-79.

4. Sarikaya S, Cimen OB, Gokcay Y, Erdem R. Pulmonary function tests, respiratory muscle strength, and endurance of persons with obesity. Endocrinologist. 2003; 13(2):136-41.

5. Rosa JS, Oliver SR, Flores RL, Milne GL, Zaldivar FP, Galassetti PR. Altered inflammatory, oxidative, and metabolic responses to exercise in pediatric obesity and type 1 diabetes. Pediatr Diabetes. 2011; 12(5):464-72.

6. Fonseca-Alaniz MH, Takada J, Alonso-Vale MI, Lima FB. Adipose tissue as an endocrine organ: from theory to practice. J Pediatr. 2007; 83(suppl 5):192-203.

7. Morrow J. Is a oxidative stress a connection between obesity and atherosclerosis? Arterioscler Thromb Vasc Biol. 2003; 23:368-70.

8. Costa D, Sampaio LMM, Lorenzzo VAP, Jamami M, Damaso AR. Avaliação da força muscular respiratória e amplitudes torácicas e abdominais após a RFR em indivíduos obesos. Rev Latino-Am Enfermagem. 2003; 11(2):156-60.

9. Frank I, Briggs R, Spengler CM. Respiratory muscles, exercise performance, and health in overweinght and obese subjects. Med Sci Sports Exerc. 2011; 43(4):714-27. 
10. Ribeiro AF, Jamami M, Elias EC. Avaliação dos efeitos do treinamento muscular inspiratório em pacientes com fibrose cística através do uso do threshold IMT. Universidade Estadual de Campinas 2006. Faculdade de Ciências Medicas Nível: Dissertação (mestrado) UNICAMP: Programa de Pós-Graduação em Saúde da Criança e do Adolescente.

11. Weiner P, Magadle R, Beckerman M, Weiner M, Berar-Yanay N. Comparison of specific expiratory, inspiratory, and combined muscle training programs in COPD. Chest. 2003; 124(4):1357-64.

12. Sociedade Brasileira de Cardiologia / Sociedade Brasileira de Hipertensão / Sociedade Brasileira de Nefrologia. VI Diretrizes Brasileiras de Hipertensão. Arq Bras Cardiol 2010; 95(1 supl.1): 1-51

13. Callegaro CC, Ribeiro JP, Tan CO, Taylor JA. Attenuated inspiratory muscle metaboreflex in endurance-trained individuals. Respir Physiol Neurobiol. 2011; 177(1):24-9.

14. American Thoracic Society/European Respiratory Society (2002) ATS/ERS Statement on respiratory muscle testing. Am J Respir Crit Care Med. 166(4):518-624.

15. Dall'ago P, Chiappa GR, Guths H, Stein R, Ribeiro JP. Inspiratory muscle training in patients with heart failure and inspiratory muscle weakness: a randomized trial. J Am Coll Cardiol. 2006; 47(4):757-63.

16. ATS statement: guidelines for the six-minute walk test. ATS Committee on Proficiency Standards for Clinical Pulmonary Function Laboratories. Am J Respir Crit Care Med. 2002;166(1):111-7.

17. Boueri FMV, Bucher-Bartelson B, Glenn K, Make B. Quality of life with a generic instrument (Short form-36) improves following pulmonary rehabilitation in patients with COPD. Chest. 2001; 119(1): 77-84.

18. Jentzsch AM, Bahmann H, Furst P, Biesalski HK. Improved analysis of malondialdehyde in human body fluids. Free Radic Biol Med. 1996; 20(2):251-256.

19. Borg GAV. Psychophysical bases of perceived exertion. Med Sci Sport Exerc. 1982; 14(5):377-81.

20. World Health Organ Tech Rep Ser. Obesity: preventing and managing the global epidemic. Report of a WHO consultation. 2000;894:i-xii, 1-253.

21. Vincent KR, Vincent HK, Braith RW, Lennon SL. Resistance exercise training attenuates exercise induced lipid peroxidation in the elderly. Eur J Appl Physiol. 2002; 87(4-5):416-23.

22. Zoppi CC, Alves AA, Silveira LR, Silva LP, Macedo DV. Efeitos dos treinamentos de endurance e intervalado em marcadores do metabolismo oxidativo, oxidantes, antioxidantes e lesão muscular. Rev Digital - Buenos Aires. 2006; 11(99). 23. Gonchar O. Effect of intermittent hypoxia on pro- and antioxidant balance in rat heart during high-intensity chronic exercise. Acta Physiol Hung. 2005; 92(3-4):211-20.

24. Neto, JMFA; Silva, LP; Macedo, DV. Proteínas de estresse "hsp70" atuam como marcadores de estresse oxidativo em ratos "wistar" submetidos a treinamento intermitente de corrida para indução de overreaching. Braz J Biomotricity 2008; 2(3):1-17.

25. Prada FJA, Voltarelli FA, Macedo DV, Mello MAR. Indicadores de estresse oxidativo em ratos submetidos ao treinamento em natação. Rev Port Cien Desp. 2004; 4(2):237-74.

26. Prada FJA, Voltarelli FA, Oliveira CAM, Gobatto CA, Macedo DV, Mello MAR. Condicionamento aeróbio e estresse oxidativo em ratos treinados por natação em intensidade equivalente ao limiar anaeróbio. R Bras Ci e Mov. 2004; 12(2):29-34.

27. Pinho RA, Soares FS, Rocha LGC, Pinho CA, Silva LA, Silveira PCL. Efeitos preventivos e terapêuticos do exercício físico sobre lesão pulmonar e estresse oxidativo induzido por bleomicina. Rev Bras Cineantropom Desempenho Hum. 2009; 11(4):415-21.

28. Radak Z, Kaneko T, Tahara S, et al. The effect of exercise training on oxidative damage of lipids, proteins and DNA in rat skeletal muscle evidence for beneficial outcomes. Free Radic Biol Med. 1999; 27(1-2):69-74.

29. Volianitis S, Mcconnell AK, Koutedakis Y, Mcnaughton L, Backx K, Jones DA. Inspiratory muscle training improves rowing performance. Med Sci Sports Exerc. 2001; 33(5):803-9.

30. Enright S, Chatham K, lonescu AA, Unnithan VB, Shale DJ. Inspiratory muscle training improves lung function and exercise capacity in adults with cystic fibrosis. Chest. 2004; 126(2):405-11.

31. Lima EVNCL, Lima WL, Nobre A, Santos AM, Brito LMO, Costa MRSR. Treinamento muscular inspiratório e exercícios respiratórios em crianças asmáticas. J Bras Pneumol. 2008; 34(8):552-8.

32. Kunikoshita LN, Silva YP, Silva TLP, Costa D, Jamami M. Efeitos de três programas de fisioterapia respiratória (PFR) em portadores de DPOC. Rev Bras Fisioter. 2006; 10(4):449-55.

33. Di Mambro TR, Figueiredo PHS, Wanderley TR, Kristki AL, Guimarães FS. Treinamento muscular inspiratório na doença pulmonar obstrutiva crônica: impacto na qualidade de vida, intolerância ao esforço e dispnéia. Fisioter Pesqui. 2007; 14(2):65-71. 
34. Silva VGDA, Amaral C, Monteiro MB, Nascimento DM, Boschetti JR. Efeitos do treinamento muscular inspiratório nos pacientes em hemodiálise. J Bras Nefrol. 2011; 33(1):62-8.

35. Dekhuijzem RPN, Folgering HTHM, Herwaarden VCLA. Target-flow inspiratory muscle training during pulmonary rehabilitation in patients with COPD. Chest. 1991; 99(1):128-33.

\section{Letícia Donato}

Endereço para correspondência - Rua: Avenida Venâncio Aires, $n^{\circ}$ 163,

Bairro: Centro, CEP: 98025-790, Cruz Alta, RS, Brasil.

E-mail: leticiadonato2010@hotmail.com

Loani Maldaner - loani_9@hotmail.com

Roberta Cattaneo Horn - rcattaneo@unicruz.edu.br

Leandro Chaves - chaves30@yahoo.com.br

Leandro de Moraes Kohl - Ikohl@unicruz.edu.br

Isabella Martins de Albuquerque - albuisa@gmail.com

Gabriela Tassotti Gelatti - gabriela.gelatti@hotmail.com

Carine Cristina Callegaro - ccallegaro@unicruz.edu.br

\section{Enviado em 30 de agosto de 2016.} Aceito em 22 de maio de 2017. 